\title{
Culturas y género: prácticas lesivas, intervenciones feministas y derechos de las mujeres ${ }^{1}$
}

\section{Cultures and Gender: Harmful Practices, Feminist Interventions and Women's Rights}

\author{
María José Guerra Palmero \\ Instituto de Estudios de las Mujeres de la Universidad de La Laguna
}

Resumen. El objetivo de este artículo, en el contexto del debate entre feminismo y multiculturalismo, es analizar el fenómeno de identidades culturales reactivas que ha operado en el caso de la interpelada práctica de la mutilación genital femenina en algunos enclaves africanos. Para ello seguiremos la peripecia de Alice Walker, la escritora y activista afroamericana, en su denuncia de esta dañina práctica cultural que atenta contra los derechos a la integridad corporal y psíquica de las mujeres. Queremos dejar de lado el sensacionalismo con que suele ser tratado en muchos medios de opinión este asunto y que contribuye a construir la alteridad de lo africano como mera barbarie y carencia de civilización. Tratamos, aquí, de analizar lo anterior en relación a la necesidad de ir construyendo una ética intercultural feminista que promueva el empoderamiento de las mujeres locales. Los contextos y los medios de intervención son relevantes para el empeño de erradicar eficazmente las prácticas culturales que violan los derechos de las mujeres al tiempo que se consolida un feminismo transnacional e intercultural.
ABSTRACT. The aim of this paper is to analyse the phenomenon of the making of reactive cultural identities in a particular and sensitive case: the female genital mutilation (FGM) in an African context. This case is framed in the debate between feminism and multiculturalism and in the controversies about the feminist transnational interventions to defend women's human rights. Alice Walker, the Afro-American writer and activist promoted an international campaign against FGM that was strongly contested by African activists. There has been a main criticism against sensationalism in the media that reduces Africa as alterity to a barbarian and uncivilised rite. We try to analyse all this in relation to the need of promoting an intercultural feminist ethics in order to fostering the process of empowerment of local women. Contexts and means are relevant if we try, first, to eradicate cultural practices that damage women's lives and rights and, also, to consolidate a transnational and intercultural feminism.

1 Este trabajo se enmarca en el proyecto I+D «Diferencias genérico-culturales y desigualdades económicas» (HUM2007-650099/FISO) del Ministerio de Educación y Ciencia de España. Es deudor, además, del haber participado, en los últimos años, en los grupos de investigación liderados por Celia Amorós y Gabriel Bello en anteriores proyectos. 
Palabras claves: identidades, feminismo, multiculturalismo, interculturalidad, mutilación genital femenina, derechos humanos.
Key words: identities, feminism, multiculturalism, interculturality, female genital mutilation, human rights.

El debate entre feminismos y multiculturalismos en, al menos, las últimas dos décadas ha planteando la necesidad de articular un diálogo intercultural feminista $^{2}$ en el contexto de la emergencia y derivas del llamado feminismo transnacional. Una de nuestras premisas ha sido recusar la idea monolítica y totalizante de la noción de cultura que descuenta la pluralidad interna y las luchas interpretativas en el seno de ésta con el fin de descalificarla y/o glorificarla sin más miramientos. El feminismo es y ha sido una disidencia cultural frente al patriarcado hegemónico. La interpelación intercultural feminista propulsa la reflexividad necesaria que cada sociedad debe practicar para erradicar los usos, prácticas e instituciones segregadoras y/o discriminadoras de las mujeres. Entendemos por culturas las maneras en las que viven mujeres y hombres y las entendemos de un modo dinámico, ya que la cultura no se puede sustraer, en las coordenadas modernas, a la aceleración y el cambio social propiciado por los procesos económicos, tecnológicos y culturales de la llamada globalización. La presión cultural que ejerce el modelo económico capitalista es un hecho innegable. Numerosos teóricos del desarrollo exigen, como condición de su posibilidad, que se quiebren las culturas tradicionales, en concreto su cemento comunitario, y que los individuos se piensen al modo del homo economicus ${ }^{3}$. Sin embargo, y a pesar de lo anterior, gran parte del multiculturalismo acrítico ${ }^{4}$ ha asumido la fijación sincrónica de la «cultura» auspiciada por la an-

2 Cf. C. Amorós, «Feminismo y multiculturalismo», en C. Amorós y A. de Miguel (eds.), Teoría feminista: de la ilustración a la globalización. De los debates sobre el género al multiculturalismo, Madrid, Minerva Ediciones, 2005, R. Cobo (ed.), Interculturalidad, feminismo y educación, Madrid, Los libros de la Catarata, 2006. M. J. Guerra, «(In)tolerancia, género y culturas: ¿cómo trazar los límites?», en Riff Raff. Revista de Pensamiento y Cultura, n. ${ }^{\circ} 27$, invierno 2005, pp. 117-128. «Algunas notas sobre feminismo global: mujeres, culturas e igualdad» en M. L. Femenías (comp.), Feminismo de París a La Plata, Buenos Aires, Catálogos, 2006. pp. 81-96. «Feminismo transnacional o feminismo global: autoritarismo, poder y pluralidad», en Democracia, Deliberación y Diferencia, Edición a cargo de Mariano C. Melero, Cuaderno Gris, Revista de Filosofía de la Universidad Autónoma de Madrid, n. ${ }^{\circ}$ 9, 2007, pp. 243-260.

${ }^{3}$ I. Adelman y C. Taft Morris, «Editorial: Development history and its implications for development theory» en World Development, vol. 25, n. ${ }^{\circ}$ 6, 1997, pp. 831-840. «Un desarrollo social significativo que ayude a romper las sociedades tradicionales, a desterrar las costumbres ancestrales y la influencia de las culturas tradicionales y conduzca a una ampliación del dominio en el que la conducta orientada hacia el mercado guíe la actividad económica», p. 839.

${ }^{4}$ Desde el feminismo liberal S. Moller Okin ha sido la teórica que más ha impulsado esta objeción. Cf. «Feminism and Multiculturalism: Some Tensions», en Ethics, vol. 108, n. ${ }^{\circ} 4$, July 1998, pp. 661-684. «Desigualdad de género y diferencias culturales», en C. Castells, Perspectivas feministas en teoría politica, Barcelona, Paidós, 1996, y Is multiculturalism bad for women?, Princeton University Press, 1999. 
tropología romántica y ha descontado, de forma equivocada, el impacto de las transformaciones sociales. Nos atrevemos a decir que el privilegio interpretativo de la sociología es total en nuestro mundo de «culturas dislocadas» ${ }^{5}$ en donde la propia reivindicación cultural es, en la mayoría de los casos, reactiva frente a la hostilidad etnocéntrica de la forma de vida dominante ${ }^{6}$ que potencia abismos de desigualdad. Appiah se pregunta, retóricamente, si no «hay una relación entre la merma del contenido cultural de las identidades y la estridencia creciente de sus reivindicaciones» ${ }^{7}$. La misma noción de cultura, un producto del romanticismo alemán en confrontación con el concepto de «civilización», ha sido asimilada a lo largo de todo el planeta como clave explicativa y reivindicativa. Para numerosos autores, el problema, como señala Appiah, es que de esta asimilación cultural, que sirve para denunciar el imperialismo cultural de Occidente, ha derivado una «ética preservacionista» de numerosas prácticas, usos e instituciones que no resisten la prueba frente al rasero normativo universalista de los derechos humanos, otra construcción tachada de etnocéntrica, a pesar de traer tras de sí una larga historia de consensos transnacionales.

El objetivo de este artículo es presentar cómo este modelo de identidades reactivas ha operado en el caso de la dañina e incapacitante práctica de la mutilación genital femenina en algunos enclaves africanos ${ }^{8}$. Nos atrevemos a tratar este doloroso asunto intentando dejar de lado el sensacionalismo con que suele ser tratado en muchos medios de opinión con el fin de alentar la percepción de lo africano como mera barbarie. La construcción social de la alteridad como barbarie e incivilización no es un buen punto de partida para propiciar ni el diálogo intercultural ni el cambio social. Se limita a estigmatizar y no quiere empeñarse en comprender para, a continuación, arbitrar las estrategias de intervención social y política más efectivas. Sólo sirve, en suma, para apuntalar los sentimientos de superioridad moral que acompañan al neoimperialismo y, que, de paso, deslegitiman la aplicación de la agenda mundial de los derechos humanos.

Nuestra aproximación a este terrible asunto, la mutilación genital femenina, intenta reflexionar sobre el reto ético de la interculturalidad desde el feminismo en su dimensión práctica. En este último sentido, apuntamos a la consideración de los límites y posibilidades de una ética y una política de la

5 Uma Narayan, Dislocating Cultures. Identities, Traditions, and Third World Feminism, New York, Routledge, 1997. Para una discusión del prejuicio culturalista, cf. U. Narayan, «Essence of Culture and a Sense of History: A Feminist Critique of Cultural Essentialism», en Hypatia, vol. 13, n. ${ }^{\circ}$ 2, pp. 86-106, 1996.

${ }^{6}$ Una discusión interesante sobre etnocentrismo la encontramos en C. Geertz, Los usos de la diversidad, Barcelona, Paidós, 1996.

7 K. A. Appiah, La ética de la identidad, Buenos Aires, Katz, 2007, p. 183.

8 Esta operación, según datos de Amnistía Internacional, se realiza en 29 países de África - con grandes diferencias entre zonas - y tres de Oriente Próximo, así como en comunidades de emigrantes en todo el mundo. En muchos de estos países está prohibida, pero la ley no se aplica. Para indagar más, véase http://www.unicef.org/spanish/protection/index_genitalmutilation.html y http://www.afrol.com/Categories/Women/FGM/netscapeindex.htm 
intervención feminista en favor de los derechos de las mujeres. Las guerras de Irak y Afganistán han señalado, además, el uso indebido de este asunto que ha protagonizado la administración Bush y cómo, en la actualidad, nada ha mejorado, más bien ha retrocedido - con el componente de aquiescencia ante las autoridades religiosas-, respecto a las condiciones de vida de afganas e iraquíes ${ }^{9}$. Para reflexionar sobre este delicado asunto, que nos enfrenta a dilemas morales enormemente difíciles, debemos alejarnos tanto de la Escila del amarillismo etnocentrista, que se contenta sólo con el ritual de la denuncia, como de la Caribdis del mirar para otra parte con que se conforma el relativismo cultural. Pero, además, debemos introducir el privilegio de la ética de la responsabilidad ${ }^{10}$ para contribuir a reducir el mal en el mundo y, por lo tanto, el como se actúa y las consecuencias concretas de la acción pasan a tener un protagonismo antes negado en función de la mera defensa, muchas veces retórica, de los principios. Sabemos que de buenas intenciones está empedrado el infierno y, en el momento actual, acumulamos experiencia suficiente cómo para plantear la importancia de los contextos y situaciones a la hora de diseñar estrategias de intervención a favor de los derechos humanos de las mujeres que sean exitosas y no refuercen, vía reactividad y efectos colaterales, la opresión que muchas de ellas están soportando.

Nadie puede dudar, por otra parte, del carácter patriarcal de las distintas culturas, la nuestra y las otras, y del desigual impacto de los procesos de ilustración, de reflexividad social, ligado a los valores universalistas de la igualdad, la libertad y la justicia en ellas ${ }^{11}$. En el seno del feminismo contemporáneo la irrupción de las diferencias, frente a un cierto etnocentrismo de partida, y la problematización de la alteridad va paralelo a la importancia que han ido cobrando perspectivas como las postcoloniales ${ }^{12}$, en el ámbito teórico, o el

9 M. L. Ferguson, «W stand for Women: Feminism and Security Rethoric in the Post 9/11 Bush Administration», Politics and Gender, vol. 1, n. ${ }^{\circ}$ 1, March, 2005.

10 R. R. Aramayo y M. J. Guerra (eds.), Los laberintos de la responsabilidad, Madrid, Plaza y Valdés, 2007. El pensamiento de Hannah Arendt inspira, en gran parte, la teorización sobre la responsabilidad política. Cf. C. Sánchez, «Hannah Arendt», en M. J. Guerra y A. Hardisson, 20 Pensadoras del siglo XX, Oviedo, Nobel, 2006.

11 Recientemente se han publicado, inspiradas en la búsqueda de las distintas «vetas de ilustración», distintas contribuciones a este asunto en C. Amorós y L. Posada (eds.), Feminismo y Multiculturalismo, Madrid, Instituto de la Mujer, 2007. Señalamos, entre otras muchas, las contribuciones de M. X. Agra, M. L. Femenías, N. Campillo, C. Sánchez, Rosa Cobo y Celia Amorós sobre el debate feminismo-multiculturalismo. En concreto, Amorós, plantea la necesidad de una ilustración intercultural en su análisis del Islam.

12 M. C. Lugones y Elizabeth Spelman, «Have We Got a Theory for You! Feminist Theory, Cultural Imperialism and the Demand for «The Woman's Voice», en N. Tuana y R. Tong (eds.), Feminism and Philosophy, Westview Press, 1995. pp. 444-507. A. Ferguson, «Resisting the Veil of Privilege: Building Bridge Identities as an Ethico-Politics of Global Feminism», en Hypatia, vol. 13, n. ${ }^{\circ}$ 3, Summer 1998, pp. 95-113. M. L. Femenías ha analizado estas aportaciones en «El feminismo colonial y sus límites», en A. de Miguel y C. Amorós (eds.), op. cit., pp. 153-213. 
activismo de las mujeres del Sur Global —expresión que con A. Jaggar preferimos a Tercer Mundo-, desde el punto de vista práctico. A este respecto, el establecer las bases justas y simétricas de un diálogo feminista intercultural, asumiendo que el feminismo y los movimientos de mujeres son siempre un fenómeno de disidencia cultural, ha sido desde los años ochenta una prioridad indiscutible. Autoras como la misma Jaggar ${ }^{13}$, Iris Marion Young ${ }^{14}$ o Seyla Benhabib ${ }^{15}$, entre otras, representan algunas de las teorizaciones ético-políticas más sugerentes en este clima de debate.

En la reflexión que presentamos hoy confrontamos el debate aludido con las prácticas lesivas que, nuestra cultura y otras culturas, se aplican de forma diferenciada a las niñas y a las mujeres. Estas prácticas en unos casos vienen avaladas por la tradición, pero, en absoluto, están al margen de la resignificación cultural en nuevos contextos sociales, económicos, políticos, etc. Como ejemplo, podríamos aludir a la desestabilización que propicia el fenómeno migratorio y su feminización. En los últimos años cabría señalar que la intervención de Ayaan Hirsi Ali en el contexto holandés ${ }^{16}$ ha sido uno de los que ha ganado más eco optando por la tolerancia cero frente a la práctica con la propuesta de una sistema de control de las familias «sospechosas» que ha levantado recelos en cuanto a vulnerar derechos constitucionales de las personas y a estigmatizar a las minorías africanas en un clima cada vez más enrarecido que es descrito por sus críticos utilizando las categorías de racismo e islamofobia. El panorama de después del 11 de septiembre de 2001, protagonizado por el terrorismo yihadista y por las guerras de Afganistán e Irak, es enormemente complicado. Civilizar este escenario de confrontación violenta es el objetivo compartido, pero la inquietud viene de la mano de la cuestión estratégica del qué hacer.

Mucho antes de la irrupción de Hirsi Ali, y a partir de la fatwa que Jomeini dictó contra Salman Rusdhie en 1989, la reflexión feminista contra los fundamentalismos se organizó tanto en el mundo árabe e islámico ${ }^{17}$ —a modo de ejemplo la WLUML - como en Europa. Mujeres contra los fundamentalismos (WAF) operó, alentando el diálogo intercultural y la solidaridad feminista, has-

13 A. M. Jaggar, «Toward a Feminist Conception of Moral Reasoning», en J. Sterba, Ethics: The Big Questions, Oxford, Blackwell, 1998, pp. 356-374.

14 I. M. Young, Democracy and Inclusion, Princeton University Press. 2000. Cf., igualmente, "Communication and the Other: Beyond Deliberative Democracy», en Intersecting Voices. Dilemmas of Gender, Political Philosophy and Policy, Princeton University Press, 1997.

15 S. Benhabib, The Claims of Culture. Equality and diversity in the Global Era, Princeton University Press, 2002.

16 Yo acuso. Defensa de la emancipación de las mujeres musulmanas, Barcelona, Círculo de Lectores, 2006.

17 La red Women living under muslim laws (WLUML) fue fundada por nueve mujeres de diferentes países: Argelia, Marruecos, Sudán, Irán, Mauricio, Tanzania, Bangladesh y constituyeron esta red de activism y reflexión en 1986. Uno de los desencadenantes fue la crisis argelina y la posterior reislamización de este país tras el fiasco de las elecciones de 1992. http://www.wluml. org/english/index.shtml 
ta 1996 y hoy es una iniciativa que se está reactivando ${ }^{18}$. Sin embargo, su eco en los medios de comunicación, globales y nacionales, es prácticamente inexistente. La denuncia de que en el Reino Unido y otros países no se vigilaba el cumplimiento de la prohibición de la mutilación genital femenina tiene una larga historia, pero muy poco eco en la opinión pública hegemónica. Organizaciones y redes como las que acabo de citar permiten no sólo centrarse en los asuntos estrella del amarillismo, sino que vigilan y denuncian también la situación global de las violaciones de los derechos humanos de las mujeres y militan contra toda forma de fundamentalismo religioso - cristiano, judío, hinduista, musulmán, etc., y trabajan en la construcción del feminismo transnacional.

La interculturalidad exige, además, que nos dejemos interpelar por las otras. Las prácticas lesivas en nuestra cultura — el caso más estudiado es la cirugía estética - son absolutamente modernas, derivadas de la utilización tecnológica al servicio de la «medicina del deseo» y se pliegan al valor social asignado en la cultura occidental a la mujer que tiene que ajustarse a un patrón corporal fijado por la delgadez (liposucciones), los pechos abundantes (implantes mamarios) ${ }^{19}$ o la juventud eterna en la piel (liftings, botox, etc.). Frente al valor tradicional de la fertilidad, que veremos ilustrado en las culturas africanas, aun deudoras del sustrato agrícola, el valor dominante en nuestra cultura consumista es el de la objetualización estética y sexual del cuerpo femenino auspiciado por la publicidad y las industrias de la belleza (moda, cosmética, etc.) que se quiere hacer compatible, no obstante, con las metas de la igualdad social y política de las mujeres. El resultado es un campo de tensiones simbólicas puesto que los estereotipos sociales, animados por los medios de comunicación, devalúan sistemáticamente a las mujeres cuando ingresan en la esfera pública. La objetualización estético-sexual de las mujeres es piedra de toque polémica para las mujeres de otras culturas y elemento utilizado por los fundamentalismos religiosos para recusar los logros de la igualdad de las mujeres. A este respecto, la crítica feminista de la llamada «tiranía de la belleza» 20 tampoco ocupa lugar mediático alguno frente a la presión consumista que alienta el Extreme Makeover y que trivializa los peligros y consecuencias de la cirugía estética. Con estas observaciones, quiero, tan sólo, llamar la atención sobre la invisibilidad social y mediática que el activismo feminista sigue teniendo. cles.htm

${ }_{18}$ Los textos a los que me refiero pueden consultarse en http://waf.gn. apc.org/arti-

Van del año 1990 al 96. Se tratan todo tipo de asuntos: escuelas religiosas, aborto, islamismo, sionismo, crímenes de honor, etc., en diferentes localizaciones nacionales y religiosas.

19 R. Millsted y H. Frith, «Being large-breasted: women negotiating embodiment», en Women's Studies International Forum, vol. 26, Issue 5, September-October 2003, pp. 455-465.

20 Por citar dos libros destacados, cf. S. Bordo, Unbearable Weight: Feminism, Western Culture, and the Body, Berkeley, University of California Press, 1993. N. Wolf, The Beauty Myth, New York, Perennial, 2002. 
En lo que sigue, vamos a analizar el tema de las prácticas lesivas contra las mujeres, poniendo como ejemplo algunas de las reacciones a la llamada mutilación genital femenina (MGF) en Africa en las últimas décadas. En esta historia se hacen presentes las tensiones que dificultan el entendimiento intercultural. El caso es que podemos analizar cómo han colisionado, por una parte, la justa denuncia feminista de lo intolerable respecto a prácticas que agreden a la integridad — corporal, psicológica, sexual,...- de las niñas y las mujeres africanas y la sensibilización del activismo pro derechos humanos respecto al factor género - básicamente en los años noventa de la mano de las Conferencias de Viena y Bejing - con, por otra parte, las acusaciones de las mujeres africanas y de otros enclaves culturales frente a cierto activismo internacional feminista cargado, según ellas, de superioridad etnocéntrica despreciativa y humillante. El debate entre universalismo y relativismo ético no creemos que sea ya relevante en este contexto, o, por lo menos, no como se ha manejado habitualmente en las discusiones sobre la diversidad cultural. Las mujeres locales a las que nos referimos son, también, activistas contra las prácticas dañinas de su propia cultura y pro derechos humanos. En consecuencia, el debate relevante se sitúa en la estimación ética-política de las estrategias de intervención y, por lo tanto, en la construcción de juicios morales compartidos — entre insiders y outsiders - inspiradores de políticas públicas - legislativas, penales, sanitarias, educativas, culturales, etc.- que sería deseable que remitiesen al diálogo feminista intercultural y al paradigma combinado de justicia como redistribución, reconocimiento y representación que recientemente ha reformulado atinadamente Nancy Fraser ${ }^{21}$.

Por último, es necesario, además, referir que el paradigma normativo de los derechos humanos junto con la corriente de pensamiento relativa al desarrollo humano ${ }^{22}$ serán ingredientes de este debate junto con las llamadas perspectivas africanas de análisis que han rechazado la utilización sensacionalista de la MGF como elemento para declarar a las culturas africanas, de forma totalizadora, como salvajes y bárbaras desde una superioridad etnocéntrica que no se justifica dada la historia (neo)colonial de Occidente en la que los valores ilustrados fueron marginados frente a los objetivos de la empresa económica y política imperialista basada en la explotación de la naturaleza y las gentes de los países del Sur Global.

21 Tras Justice interruptus. Critical Reflections on the «Postsocialist» Condition-New York, Routledge, 1997-, N. Fraser ha introducido la representación política y la necesidad de replantear los marcos de la justicia como prioridad hoy. Cf. «Mapping the Feminist Imagination: From Redistribution to Recognition to Representation», Constellations: An International Journal of Critical and Democratic Theory, 13, 3, 2005, pp. 295-307. Cf. M. J. Guerra, «Nancy Fraser. La justicia como redistribución y reconocimiento», en R. Máiz (ed.), Teorías políticas contemporáneas, Ed. Tirant lo Blanch, Valencia, 2001.

22 Para una síntesis de esta deriva, cf. M. J. Guerra y A. Hernández Piñero, «Mujeres, desarrollo y medioambiente: hacia una teoria ecofeminista de la justicia», Isegoría, 32, junio 2005, pp. 185-200. 


\section{La mutilación genital femenina: hechos e interpretaciones}

Presentar los hechos es una necesidad para lograr una mínima información acerca del tema en cuestión ${ }^{23}$. Hoy nos encontramos con que, a pesar de los intentos de erradicar la práctica, y a pesar de los numerosos países africanos que la han prohibido, la MGF sigue estando muy extendida. Se calcula que afecta a noventa millones de mujeres. Una nueva llamada a la prohibición fue hecha por una reciente conferencia internacional en Kenya ${ }^{24}$. Por otra parte, los nuevos contextos migratorios se muestran como focos, con distintos grado de virulencia, de tensiones culturales, legales y sociales en torno a la práctica ${ }^{25}$. El debate remite pues al emergente contexto de la ética y la política global. A este respecto, parece probado que cuanto más hostil es la sociedad de acogida con los inmigrantes, las prácticas culturales como la MGF, pero, también, otras como el velo musulmán, cobran una nueva significación como elementos reactivos que, en torno al cuerpo y la apariencia de las mujeres - las que ostentan la marca de la cultura y de las que depende su reproducción simbólica-, inciden en la defensa de la identidad étnica, cultural o religiosa de origen. Elizabeth Brandsfield documenta esto último al contrastar dos casos de estudio: uno referido a los EEUU, en los que la práctica se reafirma reactivamente como seña de identidad cultural - una función inexistente en su origen-, frente a Canadá en donde el grado de acogida e integración permite que las familias se permitan renunciar a la práctica entendiendo como ventajosa la situación de adaptarse a su nuevo contexto social ${ }^{26}$. Brandsfield nos invita en su análisis a entender el por qué de la práctica de MGF y, por lo tanto, a suspender nuestra primera reacción absolutamente natural de horror y escándalo. No podemos dejar de decir que lo que designamos como «violencia» viene, también, cultural, social e históricamente determinado. Nuestra percepción moral está sometida a cambios en sus registros ya que los debates sobre qué es o no tolerable no dejan de acompañarnos en una sociedad sometida a nuevos desafíos respecto a los derechos vindicados ${ }^{27}$.

23 Para más información, cf. http://www.amnesty.org/ailib/intcam/femgen/fgm1.htm

24 «New call to ban female mutilation», BBC news, World Edition, Sunday, 19 September, 2004. http://news.bbc.co.uk/2/hi/africa./3669762.stem

${ }^{25}$ Cf. A. Akpinar, «The honour/shame complex revisited: violence against women in the migration context», en Women's Studies International Forum, vol. 26, 5, September-October, 2003, pp. 425-442 y L. M. Kopelman, «Female Genital Circumcision and Conventionalist Ethical Relativism», en R. M. Tong (ed.), Globalizing Feminist Bioethics. Crosscultural Perspectives. Westview Press, 2001, pp. 219-237.

26 E. Brandsfield, «Female Genital Mutilation», MA Thesis in Gender, Anthropology and Religion. Recomendamos este trabajo para una primera aproximación crítica al tema. Se puede consultar en http://www.users.globalnet.co.uk/-lavie/fgm/dissertation.html

27 Los ejemplos pueden ser múltiples: la esclavitud, la tortura, los derechos de las mujeres o ahora, como elemento de presión de un cambio de sensibilidad moral, los derechos de los animales. 
Los argumentos que se dan a favor de la práctica son muy variados y de todo tipo - higiénicos, estéticos, morales, etc.- $\mathrm{y}$, por supuesto, son falsos ya que los riesgos sanitarios y el daño, como mutilación, en la salud psíquica, sexual y reproductiva de las mujeres está más que documentado ${ }^{28}$. La justificación religiosa de la práctica no se sostiene. Las zonas de África central islamizadas la mantuvieron ${ }^{29}$, pero países como Arabía Saudí, la cuna del Islam, la desconoce. Convive, además, con religiones animistas, con el cristianismo, e incluso en Etiopía con el judaísmo ortodoxo. En El Corán no se cita la MGF. En cambio, hay algunos hadices que la reconocen como una práctica preislámica (al-Bujârî); parece que se limitaba a la escisión del prepucio del clítoris y no aparece como una práctica obligatoria. Sin embargo, hay que tener en cuenta que en la elaboración que hacen las diferentes escuelas jurídicas de estas prácticas no habrá consenso: algunos, como por ejemplo, para la escuela malikî (actualmente en vigor en el Magreb) el jitân es una práctica obligatoria para los hombres mientras que el jifẩ es sólo recomendable (mukarimma) para las mujeres. Otros, como la escuela shâfî̀, dicen que es obligatoria ( $g a \hat{d j j i b}$ ); dicha escuela actualmente rige en Bahrein, África oriental, y Egipto ${ }^{30}$. La identificación de la MGF con el Islam ha servido, en determinados sectores, a una estrategia estigmatizadora de la religión musulmana.

No obstante, para entender la MGF, debemos interrogarnos sobre su funcionalidad social. Nos quedan claras, tras leer algunos estudios al respecto, al menos, dos cosas:

- En determinadas culturas africanas una mujer no es una mujer sin la intervención mutiladora - ésta puede ser de diversos tipos desde un mera retirada de la piel del clítoris similar a la circuncisión masculina hasta la extirpación total de los genitales femeninos en la que la «herida» se cose, la llamada infibulación-. Tiene, pues, el efecto de traumático rito iniciático, ejercido por otras mujeres dedicadas a esta tarea, con el fin de señalar el lugar de sumisión de la niña o adolescente

28 Un relato de la historia del activismo anti MGF desde los años setenta y de la «naturalización» de la práctica ligada a la presión social sobre niñas y mujeres lo encontramos en T. Levin, «Dos millones cada año ¿durante cuántos años más? Hacia la abolición de la mutilación genital femenina», en A. Gómez y J. Tally (eds.), La construcción social de lo femenino, Instituto Canario de la Mujer, 1998.

29 Es muy difícil encontrar datos concretos a no ser por los testimonios directos de algunas mujeres que, en la lucha por su erradicación social, sacan a la luz su propio caso. Así lo hizo, por ejemplo, Nawal al-Sa'dawi quien, además, tuvo la ocasión de tratar a numerosas mujeres cuando trabajó como médico rural. De su experiencia se desprende que la amputación del clítoris suele poner en peligro la vida de la niña pero, sobre todo, tiene como consecuencia directa «la frigidez sexual, a la que también contribuyen otros factores sociales y psicológicos que influyen en la personalidad y la formación de las mujeres en las sociedades árabes» (p. 60). La cara desnuda de la mujer árabe, Madrid, horas y HORAS, 1991.

30 Debo esta información a Dolores Serrano, profesora de estudios árabes de la Universidad de La Laguna. 
frente al futuro marido, pero, también, frente a las mujeres mayores. En este caso, pues, «la mujer no nace, se hace» tal como indicaba Beauvoir.

- Al extirpar el clítoris ${ }^{31}$ se pretende, además, servir al control de la sexualidad femenina como muchas otras prácticas culturales de carácter disciplinario menos sangrientas e irreversibles, pero igualmente reprobables, como las de reclusión doméstica o la vestimenta obligatoria en las más diversas localizaciones. Como ha mostrado el estudio «Female genital mutilation: implicatons for female sexualitiy» ${ }^{32}$ realizado entre estudiantes universitarias del sur de Nigeria, el efecto de control de la sexualidad es más que discutible porque las chicas «iniciadas» se sienten más maduras sexualmente y tienen antes su primera relación sexual frente a las no mutiladas. Un apunte interesante de este estudio es la dificultad de las mujeres mutiladas para entender que la práctica es lesiva, y que representa un daño irreversible, puesto que manifiestan que la harán a sus hijas. Además, desplazan su sensibilidad sexual hacia otras zonas erógenas como los pechos y sienten un cierto orgullo con respecto a su condición. La experiencia vivida de la mutilación forzada se resignifica positivamente y se convierte en un elemento de su identidad. Nussbaum a este respecto, habla de «preferencias adaptativas» y podemos señalar, además, las dificultades para distanciarse de la propia comunidad que prodiga aceptación, aunque sea a precio tan alto.

O sea, la práctica representa la «marca» que convierte a la niña o a la chica en «una de los nuestros». La cohesión social del grupo se juega en la práctica junto a que el no haberla padecido supone perder valor objetivo en el mercado matrimonial. La pseudojustificación de la práctica abunda en el valor que estas sociedades africanas -Edos, Aniomas, Itshekiris, Isokos y Urhobos son las correspondientes al sur de Nigeria- dan a la fertilidad junto con el miedo masculino a la insaciabilidad sexual de las mujeres. La MGF ha sido un tema oculto y velado, un asunto de mujeres y para mujeres, que remite, incluso, a una supuesta «solidaridad» entre madres e hijas. Una madre que no exponga a su hija a la extirpación en determinadas comunidades es considerada una mala madre que no cumple con sus deberes y, por tanto, se sentirá culpable de no dar cabida a su hija para una vida social normalizada con éxito matrimonial.

¿Por qué remitimos a estas consideraciones? Porque la principal critica al activismo feminista internacional por parte de las mujeres africanas ha sido el de haber descontextualizado la práctica para enfatizar su brutalidad y salva-

31 Para una descripción de las variantes de la práctica cf. E. Dorkenoo, Cutting the Rose. Female Genital Mutilation: The Practice and its Prevention, 1994.

32 S. Dibieamaka Nwajei y A. Iwesim Otiono, «Female genital mutilation: implicatons for female sexualitiy», en Women's International Forum, vol. 26, Issue \&, November-December 2003, pp. 575-580. 
jismo - no hay que subestimar el hecho de que se realiza sin consentimiento a niñas y adolescentes - y trasladarla a todo lo africano sin matices para condenarlo sin paliativos. El desafío suena así: ¿por qué tanto escándalo en torno a la MGF y tan poco respecto a las condiciones de vida de las mujeres africanas subsaharianas cuya expectativa de vida es de 35 años, su situación nutricional, sanitaria y educativa desastrosa y su equiparación civil y política inexistente? El llamado «síndrome de la misionera» se ejemplifica así desde la superioridad etnocéntrica de las que son llamadas a salvar a las «ignorantes y sometidas» mujeres del Tercer Mundo. A continuación, ejemplificaremos este asunto de la mano de la intervención que la feminista negra y escritora Alice Walker ha tenido en esta historia ${ }^{33}$.

\section{Intervenciones feministas deslocalizadas y efectos colaterales}

La alarma al respecto de la MGF cundió en los años setenta, en la década de la ONU para las mujeres, en la que esto que llamamos feminismo internacional empezó a fraguarse. La ayuda al desarrollo y, después, una cierta inclinación hacia la necesidad de atender a las mujeres junto con los enormes problemas sanitarios provocados por la práctica — desde muerte a infecciones, pasando por esterilidad o partos difíciles - , hicieron que se propagara la alerta roja. La demanda de atención sintonizó con las transformaciones del feminismo urgido por redefinir, incluyendo a la pluralidad de situaciones de las diversas mujeres, su proyecto liberador respetando las diferencias y librándose de su condición de producto social de clase media educada y blanca europea y estadounidense.

En este contexto, la irrupción del feminismo negro o womanism alentado por Alice Walker, entre otras, fue decisiva ${ }^{34}$. Frente al canon feminista blanco se reivindicaba la experiencia de triple marginación vivida por las mujeres negras - raza, clase y sexo-y se recuperaban las claves culturales afroamericanas ligadas a las relaciones entre mujeres y, especialmente, entre madres e hijas. Walter, que encarnó este justo desafío y exigió el compromiso antirracista del feminismo norteamericano, va a sufrir un fuerte revés cuando invocando la solidaridad feminista y negra atienda al problema de la MGF. Su desembarco en África no va a ser comprendido por las mujeres africanas que van a tildar su visión — expresada en Possesing the Secret of the Joy ${ }^{35}$ y War-

33 Cf. la consideración que C. Alborch hace de la importante escritora y activista negra en Libres. Ciudadanas del mundo, Madrid, Aguilar, 2004.

${ }_{34}$ M. J. Guerra, «La intersección género-raza. El ejemplo del feminismo negro norteamericano», en Teoría feminista contemporánea. Una aproximación desde la ética, Madrid, Ed. Complutense, 2001.

35 London: Jonathon Cape, 1992. Seleccionamos una breve nota sobre Alice Walker (Eatonton, Georgia, 1944). Poeta, novelista, activista del Movimiento por los Derechos Civiles en su país. Por su novela El color púrpura fue acreedora del Premio Pulitzer en 1983. Entre 
rior Marks: Female Genital Mutilation and the Sexual Blinding of Women ${ }^{36}$ - de imperialismo cultural y de falta de empatía con la situación de las mujeres en las sociedades afectadas ${ }^{37}$ _. El tono de Walker es de acusación y denuncia iracunda. No hay tiempo ni para comprender, ni para dialogar puesto que estamos ante una urgencia moral insoslayable. Walker estigmatiza a las mujeres mayores de los grupos culturales que las practican no entendiendo cómo su visión es un producto social, las tilda de ignorantes y bárbaras y ellas, por contra, se cierran en banda y se sientes insultadas y humilladas. Aun cuando, desde la perspectiva de enarbolar los correctos principios morales Walker se crea justificada, que lo está, la estrategia es errónea y según S. Mekuria ha traído más mal que bien.

Intentemos aclarar algo esta resbaladiza cuestión. Desde una estimación ética de las consecuencias podemos advertir que, quizás, el bien que han traído intervenciones como la de Walker ha revertido en la propia comunidad occidental al transformar la visión del activismo proderechos humanos incorporando la perspectiva de género ${ }^{38}$ y esta influencia ha tenido una importancia decisiva en las prioridades de las ONGs durante los años noventa y sobre los objetivos planteados por la ONU en las distintas conferencias mundiales sobre la mujer. Este proceso se incardina en el dinamismo a favor de la mundialización de los derechos humanos y de las demandas de desarrollo humano en las que el no estar sometido a violencias o abusos son determinantes tal y como apunta Martha Nussbaum en Las mujeres y el desarrollo humano ${ }^{39}$.

Sin embargo, desde el lado africano, tenemos que anotar en el «debe» el mal. En el debate feminista, y ligado a las organizaciones de mujeres locales, se desecha el blindaje que el relativismo cultural ha promovido para defender la diversidad cultural al margen de su estimación moral. La opción feminista ante esto es respetar la diversidad cultural benigna — como «casos benignos» propone Z. Bauman la conservación de las lenguas $40 \_$, pero objetar la diversidad

otras obras ha publicado The Temple of My Familiar (1989), Possesing the Secret of Joy (1992) y By the Light of My Father's Smile (1998). Ocupa un lugar destacado entre los escritores estadounidenses contemporáneos.

36 A la vez un libro y un documental junto a la directora británica de origen indio Prathiba Parmar. La crítica a Walker señala que «Tashi, es sexualmente disfuncional, asesina psicótica y mártir, como símbolo de todas nosotras, una víctima sin capacidad de acción ni voluntad positiva». Esto lo escribe Salem Mekuria en «Female Genital Mutilation in Africa: Some African Views», en ACAS Bulletin, n. ${ }^{\text {ss }}$ 44/45 Winter/Spring 1995.

37 Para otra visión crítica, cf. L. King, «Warrior Marks: Female Genital Mutilation and the Sexual Blinding of Women», en African American Review, Fall, 1997.

38 «La ceguera de género del marco internacional de los derechos humanos ha tenido como consecuencia que enormes violaciones de los derechos de las mujeres hayan sido a menudo ignorados y que la discriminación estructural de las mujeres no haya sido desafiada», Amnesty International, 1998, 5. Citado por E. Brandsley en documento citado.

39 M. Nussbaum, Las mujeres y el desarrollo humano, Barcelona, Herder, 2002.

40 Z. Bauman, Comunidad. En busca de seguridad en un mundo hostil, Madrid, S. XXI, 2003, pp. 162-3. 
cultural «maligna» que colisiona con la integridad, la libertad y la igualdad de las mujeres. En este sentido, opciones como la de John Gray invitando tan sólo a la tolerancia cultural a la antigua usanza preliberal e igualando universalismo moral como imperialismo cultural estadounidense en los tiempos de la globalización son, desde un punto de vista feminista, enormemente discutibles ${ }^{41}$. Tan discutibles como las llamadas indiscriminadas a preservar cualquier «variedad cultural» como valiosa, como si se tratase de biodiversidad, sin estimar éticamente sus consecuencias sociales. El «caso maligno» de la MGF propicia entre mujeres locales feministas africanas y outsiders occidentales - Walker no puede dejar de ser estadounidense aunque se reclame afroamericana - un consenso sobre la deseabilidad de la erradicación de la práctica. Pero, el pasar del consenso feminista sobre lo que queremos erradicar a cómo logramos erradicarla como práctica cultural es aquí el núcleo del debate, del disenso sobre estrategias de intervención. Los medios son susceptibles tanto de valoración moral como de evaluación respecto a su eficacia. Cuando se valora la acción de Walker, y otras similares, desde algunas voces insertas en el contexto africano ${ }^{42}$ se le acusa de un triple reduccionismo:

- Reducir África y sus cincuenta y tantos países y cientos de culturas a un lugar «tradicional» fuera de la historia y opuesto al moderno e ilustrado Occidente.

- Reducir a las mujeres africanas a sus genitales. Una de las constantes de las críticas que se dirigen a este respecto a Occidente es su obsesión por la sexualidad como aspecto privilegiado de la existencia humana. La moral sexual es siempre un asunto candente.

- Reducir, por último, los diferentes tipos de escisión a la más extrema que es la infibulación para cargar los tintes sensacionalistas.

$\mathrm{Al}$ descontextualizar la práctica de su contexto social, económico, cultural y, sobre todo, de las relaciones de dominio neocolonial por parte de Occidente, la reacción no se hará esperar y las críticas arreciarán, amargamente, contra la buena voluntad de las activistas extranjeras. En vez de ir en la buena dirección, desde el punto de vista de la responsabilidad y la eficacia de la intervención, habremos ido en la mala. La reacción defensiva identitaria tiene la consecuencia de reforzar la práctica.

El respeto por las mujeres de otros enclaves culturales pasa hoy por el diálogo intercultural que sólo es posible si apoyamos el empoderamiento y el acti-

41 Cf. J. Gray, «¿Por qué no sabemos aún qué significa ser moderno?», en Al Qaeda y lo que significa ser moderno, Barcelona, Paidós, 2004, p. 156.

42 S. M. James y C. C. Robertson (eds.), Genital Cutting and Transnational Sisterhood: Disputing U.S. Polemics, Urbana, IL, University of Illinois Press, 2002. El reduccionismo citado lo sintetiza J. Van Allen en la reseña de la obra aludida titulada «Good Intentions» en http://www.wellesley.edu/WomensReview/archive/2002/12/highlt.html 
vismo de las mujeres locales ${ }^{43}$ y confiamos en su autonomía, abierta al diálogo, y no al dirigismo exterior, para intervenir de la manera más adecuada posible con tal de eliminar o minimizar lo que se ha llamado la resistencia cultural de hombres y de mujeres a los cambios emancipadores ${ }^{44}$. En el análisis feminista, sobre todo, hay que tener en cuenta que el contexto social desigual entre los sexos es el que refuerza y otorga vigencia a la práctica lesiva. Si la supervivencia de las mujeres africanas y el alivio de sus familias no dependieran del mercado matrimonial, sería más fácil cuestionar una práctica dirigida «a dar más valor» a la niña o a la púber en tal contexto mercantil. Para la contextualización de la práctica no es importante tan sólo la «cultura», sino cómo ésta queda delimitada y moldeada por requerimientos económicos, sociales y políticos. Aludir tan sólo a la MGF como mera práctica cultural lesiva ajena a otros factores alimenta el sensacionalismo y el escándalo, refuerza el etnocentrismo occidental que construye la alteridad como barbarie y, lo que es peor, alienta las reacciones identitarias que se atrincheran en la defensa de las prácticas denunciadas. El efecto «rebote» es que se revitalizan prácticas dañinas y opresivas que se estaban abandonando al hilo de los cambios sociales igualitarios.

El hecho es que muchas mujeres africanas se rebelan frente a esta identificación con la muda víctima. Ellas se perciben a sí mismas como agentes y resistentes en unas condiciones de injusticia internacional que desangran, literalmente, al continente a través del hambre, la pobreza o esa huida, muchas veces suicida, que es la emigración. Otra recontextualización importante, relativo a este último asunto, ha sido la batalla legal para que el motivo de huir de la MGF sea fundamento de la concesión de asilo político con el fin de lograr el estatuto de refugiada ${ }^{45}$. Los efectos de lo anterior, también, han sido ambivalentes: de un lado una profundización positiva en la perspectiva de género en la defensa de los derechos humanos, de otro lado, el rechazo político y social desde los países africanos por la imagen bárbara y totalizadora que se da de ellos en los medios de comunicación ${ }^{46}$. La estrategia que podemos llamar «cruzada», tan estadounidense ella, contra la MGF es la que se recusa.

43 Cf. A. Donchin, «Converging Concerns: Feminist Bioethics, Development Theory, and Human Rights», Signs, vol. 29, n. ${ }^{\circ}$ 2, 2003, pp. 299-324.

44 M. Nussbaum, «Women's Education: A Global Challenge», Signs, Journal of Women in Culture and Society, vol. 29, n. ${ }^{\circ} 2,2003$, pp. 325-355. En este artículo se discute el asunto de la «resistencia cultural».

45 Aminata Diop de Mali rechazó la operación y fue la primera mujer, en 1990, en solicitar en Francia asilo político usando a la Convención de Ginebra como fundamento de su petición. El juez se negó a garantizarle el derecho de asilo por falta de pruebas. No fue extraditada y estableció un precedente. Canadá fue el primer país en garantizar el estatuto de refugiadas a las mujeres amenazadas. Cf. T. Levin, art. cit., pp. 80-81.

46 Hemos encontrado información en Internet sobre polémicas en los Estados Unidos al respecto acusando a líderes feministas como Gloria Steinem o incluso a Hilary Clinton de encubrir casos fraudulentos. Es difícil demarcar la legítima lucha feminista de la vocación de ciertos medios de comunicación por la sensación y el escándalo que ha podido condicionar mucho la percepción del mensaje feminista de apoyo a las mujeres que se rebelan como la MGF. 
El tratamiento sensacionalista, que se centra en la anécdota y el caso, no se concilia con el análisis feminista de la opresión de género como un continuo que va desde el aborto selectivo o infanticidio femenino en países asiáticos hasta la MGF en África, desde la malnutrición sistemática de las niñas hasta la carencia de asistencia sanitaria, desde el analfabetismo femenino hasta la sobrecarga explotadora de trabajo dentro y fuera del hogar, desde la falta de libertad sexual y reproductiva, hasta la falta de derechos políticos, etc. Por otra parte, la opresión de género suma, en Occidente y en las élites del Tercer Mundo, esto es, en contextos de opulencia consumista, nuevos fenómenos a las lacras de la desigualdad basadas en el valor social concedido a la belleza, la delgadez y la juventud femenina.

Desgraciadamente, la ideología patriarcal no desaparece en las sociedades llamadas desarrolladas, sino que se reformula en diferentes registros culturales y sociales y se muestra aliada de las nuevas tecnologías médicas y de la nueva ascética femenina que conduce a la debilitación y la impotencia de las mujeres. En los distintos contextos, la objetualización sexual y reproductiva de las mujeres está servida. Esto último no significa decir que todo es lo mismo por qué, obviamente, no lo es: el «maligno caso», por seguir con la terminología de Bauman, del que estamos tratando revela la violencia ritualizada y normalizada descargada sobre niñas y adolescentes que no consienten, ni deberían consentir nunca, a la práctica. Pero hacer de la MGF piedra de escándalo mediático ha resultado una mala estrategia para la acción local africana. Ha servido para alentar una recepción distorsionada del feminismo como insensible producto imperialista. El hecho es que es más fácil erradicar las prácticas culturales si no son funcionales al grupo social tanto en el plano material y económico, como en el simbólico. La realidad sociopolítica de las relaciones de género es lo que debemos focalizar y, en este sentido, de lo que se trata es de fomentar el empoderamiento de las mujeres africanas y de luchar por condiciones socioeconómicas de igualdad que les permitan, no sólo sobrevivir al hambre y la enfermedad y no ser mutiladas brutalmente, sino cotas importantes de desarrollo e independencia. Junto a la necesaria prohibición legislativa - las leyes disuaden y educan - que deje claro que los Estados la recusan, aunque no debemos olvidar la condición precaria del estado en África, se deben fomentar estrategias educativas generales y particulares. Por ejemplo, ha resultado útil entrenar en otras tareas sanitarias a las extirpadoras - una especie de reconversión profesional — para que así no vean disminuido su status grupal y su ascendiente entre las mujeres que es lo que las motiva material y simbólicamente para ejercer su siniestro «oficio» al servicio del patriarcado. La intervención de la feminista occidental, aunque sea negra y afroamericana como Walker, debe estar sometida a una autocrítica vigilante dada la terrible asimetría que divide el mundo de Norte a Sur y que, desgraciadamente, hace sospechosa a la necesaria mundialización de los derechos humanos porque lo que ha sido globalizado, sin lugar a dudas, es la 
explotación económica y el desprecio de los otros y otras culturalmente diferentes. Reconocer lo anterior no debe disuadirnos del objetivo de contrariar a la «tradición» y objetar las prácticas lesivas a la vez que mostrar el sinsentido de su mantenimiento. Cada niña que pueda ser afectada nos dirige la siguiente acusación: «tú, ¿qué hiciste para evitarlo?». La urgencia ética de este problema no puede ser descontado del análisis. Los dilemas morales ligados al denominado «derecho de intervención» en el contexto transnacional nos acucian y no son nada fáciles de resolver. No obstante, al feminismo como movimiento social internacionalizado se le exige un compromiso contra la desigualdad Norte/Sur y una apuesta por la justicia global. Una orientación es que es mejor acusar primero a las estructuras de dominación que a las mujeres atrapadas en ellas y que actúan como defensoras y guardianas de los imperativos patriarcales. Por otra parte, combatir el antifeminismo reinante con inteligencia es un reto ante el resurgir de neocomunitarismos culturales y religiosos obsesionados con reforzar la sumisión de las mujeres en todo el planeta.

La dirección estratégica del feminismo transnacional exige conciliar la lógica de los principios morales, que orientan y mandan normativamente salvaguardar la integridad y libertad de las mujeres, con estimaciones éticas de las consecuencias de las estrategias de intervención porque nuestro objetivo, aquí y allí, es acercarnos a la eliminación de las distintas formas de violencia (externas e internalizadas) que alientan las prácticas culturales del patriarcado. Otro objetivo de nuestro trabajo es el de ir articulando, sobre el análisis de casos concretos, las indicaciones del contextualismo postconvencional que Benhabib proponía frente al formalismo ajeno al análisis concreto al modo Kohlberg-Habermas ${ }^{47}$, así como el universalismo interactivo ${ }^{48}$ como elemento clave de una ética feminista intercultural. Ninguna cultura puede sustraerse a la criba del análisis ético-feminista para detectar las específicas modulaciones de la violencia contra la integridad, la libertad y la igualdad de las mujeres. La autocrítica y la reflexión sobre los diversos contextos de intervención feminista como radicalización ilustrada no están reñidas con la denuncia sin paliativos de lo intolerable. Intentemos, en la medida de lo posible, desactivar las reacciones identitarias que refuercen la opresión de género. Tenemos, en suma, la obligación de elegir los medios adecuados para erradicar las prácticas culturales dañinas que atentan contra los derechos humanos de las mujeres y de todos.

47 Cf. S. Benhabib, «El otro generalizado y el otro concreto: la controversia Kohlberg-Gilligan y la teoría feminista», en S. Benhabib y D. Cornell, Teoría feminista y teoría crítica, Valencia, Alfons el Magnànim, 1990, pp. 119-149, y «The Debate over Women and Moral Theory Revisited», en Situating the Self, Routledge, 1992.

48 Cf. C. Sánchez, «Seyla Benhabib: hacia una universalismo interactivo», en R. Máiz (ed.), Teorías políticas contemporáneas, Ed. Tirant lo Blanch, Valencia, 2001. 